has improved the possibility of controlling disease activity and slowing the pro gression of joint damage. However their impact on work participation is unclear. Objectives: To assess the effect of biological and tsDMARDs versus conven tional treatments in patients with CIAs on work outcomes : employment, presenteeism and absenteeism

Methods: A systematic review of the literature using Pubmed-Medline and the Cochrane library was performed until January 2017. All randomised controlled trials (RCT) and controlled cohorts (CC) comparing work outcomes in patients with rheumatic diseases such as rheumatic arthritis (RA), ankylosing spondylarthris (AS) and psoriatic arthritis (PsA) treated with biological or tsDMARDs versus conventional therapies were selected. Statistical analysis determined in each study effect size (ES) or odds-ratios (OR) as appropriate to assess the magnitude of treatment effect. Pooled ES and OR were computed by meta-analysis. A random effect model was used in case of heterogeneity.

Results: Thirty six RCTs and eight CCs were analysed ie 12769 patients with conventional treatment and 19875 patients with bDMARD or tsDMARD (4619 Infliximab, 4629 Etanercept, 3872 Adalimumab, 670 Golimumab, 2101 Certolizumab, 691 Abatacept, 444 Sirukumab, 1668 Baricitinib, 672 Tofacitinib, 365 Sarilumab, 444 Sirukumab etc); 34 studies included 30423 patients with RA, 7 studies included 1496 patients with AS and 3 studies included 725 patients with PsA.

This meta-analysis showed in patients treated by bDMARD vs conventional treatment:

- a significant decrease of accumulated missed workdays at week 24: ES -0.34 IC95\%[-0.6; - 0.08] and at week 52: ES -0.04 IC95\% [-0.29;0.2],

- a significant decrease of patients loosing hours due to CIAs: RR 0.63 IC95\% [0.48; 0.83],

- a significant improvement in VAS productivity: ES - 1.81 IC95\%[-2.61; -1.01],

- For the employment loss, the positive effect of bDMARDs was nearly significant: OR 0.60 IC95\% [0.33; 1.09$]$.

Conclusions: Despite the heterogeneity of the data, this meta-analysis showed the beneficial effect of bDMARDs on both absenteeism and presenteeism in CIAs. Thus the high cost of biologic agents could be partly balanced with savings in indirect costs.

Disclosure of Interest: C. Traverson: None declared, A. tubery: None declared, C. Hua Consultant for: Pfizer, BMS, abbvie, F. Barchechath-Flaisler Consultant for: Roche Pharmaceuticals, C. Lukas Consultant for: Abbvie, BMS, Celgene, Janssen, MSD, Novartis, Pfizer, Sanofi, Schering, Roche- Chugai, UCB, B. Combe Grant/research support from: Pfizer, UCB, Consultant for: Abbvie, BMS, Janssen, Lilly, MSD, Novartis, Pfizer, Roche-Chugai, UCB, Speakers bureau: BMS, Janssen, Lilly, MSD, Pfizer, Roche-Chugai, UCB, J. Morel Consultant for: Abbvie, BMS, Celgene, Janssen, Medac, MSD, Novartis, Pfizer, Sanofi, Schering, Roche- Chugai, UCB, C. Gaujoux-Viala Grant/research support from: Pfizer, Consultant for: Abbvie, BMS, Celgene, Janssen, Medac, MSD, Nordic Pharma, Novartis, Pfizer, Sanofi, Roche- Chugai, UCB

DOI: 10.1136/annrheumdis-2018-eular.4839

\section{FRI0107 FIVE SUCCESSFUL PREGNANCIES WITH ANTENATAL ANAKINRA EXPOSURE}

C.J.F. Smith ${ }^{1}$, C. Chambers ${ }^{2} .{ }^{1}$ Rheumatology, Allergy, Immunology, ${ }^{2}$ Pediatrics, University of California San Diego, La Jolla, USA

Background: Current recommendations are to discontinue the interleukin-1 (IL1) inhibitor anakinra prior to pregnancy given lack of safety evidence. ${ }^{1} \mathrm{~A}$ total of 39 previous exposed pregnancies have been documented in the literature, and two resulting cases of fetal renal agenesis have been described. ${ }^{2,3}$

Objectives: Our aim is to add to the limited existing data on IL-1 inhibitor use in pregnancy.

Methods: Data were obtained from the Organisation of Teratology Information Specialists (OTIS) Autoimmune Disease in Pregnancy Project, a prospective cohort study of pregnancy outcomes in the U.S. and Canada. Eligible women enrolled prior to 19 weeks' gestation between 2004 and 2017. Women who consented to participate were interviewed two to three times during pregnancy with a standard questionnaire regarding medical history, exposures during pregnancy, and demographic characteristics. Outcomes were obtained by maternal interview and medical record abstraction.

Results: Five pregnancies with anakinra exposure were identified, all resulting in full-term singleton live births, mean gestational age at delivery of 38.9 weeks, with no major or long-term infant complications. Three maternal subjects used anakinra for adult-onset Still's disease (AOSD) and two for juvenile idiopathic arthritis (JIA). One mother was diagnosed with AOSD during pregnancy, and anakinra was started at 20 weeks' gestation. All other subjects had a known diagnosis prior to pregnancy, and four subjects used the medication into the third trimester. All maternal subjects used $100 \mathrm{mg}$ dosing of anakinra, four with daily use, one with weekly use. For all subjects who discontinued anakinra, some amount of steroid medication was necessary for treatment of disease flare. Two subjects developed oligohydramnios, one also with pregnancy-induced hypertension. Two women had caesarian sections, one medically-indicated and one scheduled. One infant had low birth weight, but follow-up records indicated normal adjusted weight at one year. Three women successfully breastfed their infants, at least two of whom continued anakinra while breastfeeding.

Conclusions: Anakinra was used successfully in five full term pregnancies. Two subjects developed oligohydramnios, however. With the two previous reported cases of fetal renal agenesis, a process leading to low fluid levels, and the fact that maternal hyperthermia has been previously linked to fetal renal anomalies, ${ }^{4}$ it is worth further exploring the potential link between anakinra use, uncontrolled maternal febrile disease, and fetal outcomes. These data add to the limited knowledge regarding antenatal anakinra use and support its use in pregnant women without access to effective alternative therapies that have a larger volume of reas suring safety data.

\section{REFERENCES}

[1] Götestam Skorpen C, et al. The EULAR points to consider for use of antirheumatic drugs before pregnancy, and during pregnancy and lactation. Ann Rheum Dis 2016;75:795-810.

[2] Chang Z, et al. Anakinra use during pregnancy in patients with cryopyrinassociated periodic syndromes (CAPS). Arthritis Rheumatol 2014;66:3227-32.

[3] Youngstein T, et al. International multi-centre study of pregnancy outcomes with interleukin-1 inhibitors. Rheumatology (Oxford). 2017;56:2102-2108.

[4] Abe K, et al. Maternal febrile illnesses, medication use, and the risk of congenital renal anomalies. Birth Defects Res A Clin Mol Teratol 2003;67:911-8.

Disclosure of Interest: None declared

DOI: 10.1136/annrheumdis-2018-eular.1849

\section{FRI0108 GOLIMUMAB IMPROVES WORK PRODUCTIVITY AND ACTIVITY AND QUALITY OF LIFE IN PATIENTS WITH RHEUMATOID ARTHRITIS (RA), PSORIASIS ARTHRITIS (PSA) AND AXIAL SPONDYLOARTHRITIS (AXSPA): INTERIM RESULTS FROM A NON-INTERVENTIONAL STUDY IN AUSTRIA (GO ACTIVE)}

C. Dejaco ${ }^{1}$, T. Mueller $^{2}$, O. Zamani ${ }^{3}$, U. Kurtz ${ }^{4}$, S. Egger ${ }^{5}$, J. Resch-Passini $^{6}$, A. Totzauer $^{7}$, W. Eisterer ${ }^{8}$, B. Yazdani-Biuki ${ }^{9}$, T. Schwingenschloegl ${ }^{10}$, P. Peich ${ }^{11}$, A. Kraus ${ }^{12}$, G.W. Naerr ${ }^{13}$, V. Rickert ${ }^{13}$. ${ }^{1}$ Medical University Graz; ${ }^{2}$ Private Office, Graz; ${ }^{3}$ Rheuma Zentrum Favoriten, Vienna; ${ }^{4}$ Rheuma Praxis Dr. Kurtz, Gleisdorf, ${ }^{5}$ Ordination Gesundheitsquadrat, ${ }^{6}$ Rheuma-Zentrum Wien-Oberlaa GmbH, Vienna ${ }^{7}$ Private Office Dr. Anna Totzauer, Gmünd; ${ }^{8}$ Klinikum-klagenfurt am Wörthersee, Klagenfurt, ${ }^{9}$ Dr. Schrenk and Dr. Yazdani-Biuki OG -Gruppenpraxis, Fürstenfeld; ${ }^{10}$ Private Office Dr. Thomas Schwingenschloegl, Wiener Neudorf, ${ }^{11}$ Private Office Prim. Univ. Doz. Dr. Peter Peichl, Vienna; ${ }^{12}$ Private Office Dr. Angelika Kraus, Melk; ${ }^{13}$ Merck Sharp and Dohme Gmbh, Ares Tower, Donau-City-Straße 11, 1220 Vienna, AUSTRIA, Vienna, Austria

Background: Golimumab has shown clinical efficacy and tolerability within its clinical trial program. No systemic outcome data regarding patient-reported outcomes and health economic parameters reflecting the real world use of golimumab in Austria are currently available.

Methods: Go Active is a prospective, non-interventional, multi-centre study in Austria. The impact of golimumab therapy on work productivity and activity (WPAI) and quality of life (RAQoL for RA patients, AsQoL for axSpA patients, PsAQoL for PsA patients) is assessed by using patient reported outcomes. Patients (target recruitment: $n=320$ ) are followed up to 2 years. For the current interim analysis (data cut-off: 18DEC2017) changes in the primary endpoint from baseline to month 3 were analysed in 167 patients.

Results: 167 patients are included in the current analysis ( 74 patients with RA, 49 patients with axSpA, and 44 patients with PsA). At study entry, most patients were biological-naïve and employed.

Median age at registration was 52 years (patients with RA: 57 years, patients with axSpA: 41 years, and patients with PsA: 44 years). Almost 2/3 of patients were female (84\% of patients with RA, $37 \%$ patients with axSpA, and $55 \%$ of patients with PsA). Most patients were biological-naïve at study entry $(77 \%$ of all patients $73 \%$ of patients with RA, $80 \%$ of patients with axSpA, and $82 \%$ of patients with PsA). $42 \%$ of patients were not employed (58\% of patients with RA, $29 \%$ of patients with axSpA, and $30 \%$ of patients with $\mathrm{SpA}$ ); $14 \%$ due to incapacity for work ( $12 \%$ of patients with RA, $21 \%$ of patients with axSpA, and $16 \%$ of patients with $\mathrm{SpA}$ ) and $54 \%$ due to age-related pension ( $60 \%$ of patients with RA, $21 \%$ of patients with axSpA, and $69 \%$ of patients with $\mathrm{SpA}$ ). Most of the patients, who worked for pay, worked full time. 159 of all patients and 66 of employed patients completed the WPAI questionnaire at baseline and after 3 months. Overall work productivity improved by -33 ( -40 for patients with RA, and -31 for patients with axSpA and PsA) and activity impairment by -30 ( -40 for patients with RA and 
axSpA, and -20 for patients with PsA; figure 1). Quality of life improved by -5 for patients with axSpA and PsA, and by -7 for patients with RA.

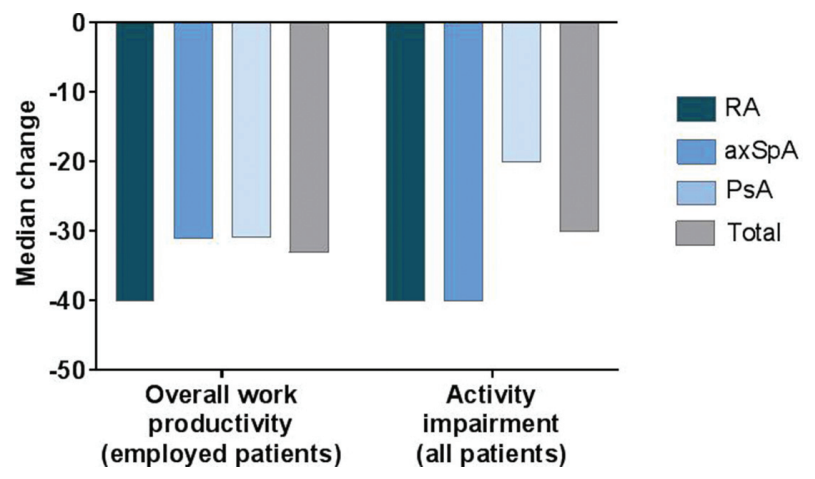

Abstract FRI0108 - Figure 1. WPAI questionnaire - Changes from baseline after 3 months of golimumab treatment

Conclusions: This interim analysis shows that golimumab treatment is effective in improving work productivity, daily activities as well as quality of life already within the first 3 months of treatment in RA, axSpA and PsA patients.

Disclosure of Interest: C. Dejaco Consultant for: Consulting fees from Merck Sharp and Dohme, Paid instructor for: Remuneration form Merck Sharp and Dohme, Speakers bureau: Merck Sharp and Dohme, T. Mueller: None declared, O. Zamani: None declared, U. Kurtz: None declared, S. Egger: None declared, J. Resch-Passini: None declared, A. Totzauer: None declared, W. Eisterer: None declared, B. Yazdani-Biuki: None declared, T. Schwingenschloegl: None declared, P. Peichl: None declared, A. Kraus: None declared, G. Naerr Employee of: Merck Sharp and Dohme Ges.m.b.H, V. Rickert Employee of: Merck Sharp and Dohme Ges.m.b.H

DOI: 10.1136/annrheumdis-2018-eular.2411

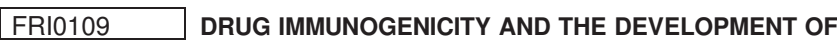 PARADOXICAL ADVERSE EVENTS WITH BIOLOGIC THERAPIES}

C. Ancuta $^{1,2}$, C. Pomirleanu ${ }^{1}$, R. Paiu ${ }^{1}$, G. Strugariu ${ }^{1}$, L. Petrariu ${ }^{1}$, E. Ancuta ${ }^{3}$, A. Chiriac ${ }^{4}$, R. Chirieac ${ }^{5} .{ }^{1}$ Clinical Rehabilitation Hospital; ${ }^{2}$ University of Medicine and Pharmacy Grigore T Popa lasi; ${ }^{3}$ Elena Doamna Clinical Hospital; ${ }^{4} \mathrm{CMI}$ Dermatology Nicolina Center, ${ }^{5}$ Sanocare Medical and REsearch Center, lasi, Romania

Background: Defined as de novo or exacerbation of pathologies that usually responds to biologic agents, paradoxical reactions encompass for a wide spectrum of manifestations (cutaneous, intestinal, ocular) reported during biological therapy (TNF and non-TNF medications) regardless of the underlying rheumatic or non-rheumatic disorder.

Objectives: To assess drug levels (DL) and anti-drug antibodies (ADA) in patients with paradoxical reactions (de novo or exacerbation of psoriasis, uveitis, Crohn's disease) induced by biological drugs (bDMARS) in Rheumatoid Arthritis (RA), Ankylosing Spondylitis (AS) and Psoriatic Arthritis (PsA).

Methods: We performed a retrospective observational study in cohorts of consecutive RA (267), PSA (65) and AS (145) undergoing TNF (i-TNF) or non-TNF biologics according to the local recommendations for initiation and monitoring, attending a single academic rheumatology department.

All patients were assessed at every 24 weeks months for disease activity and outcomes; supplementary, drug immunogenicity (ADA and DL) was systematically evaluated in patients developing paradoxical adverse events.

DL and ADA were measured with an ELISA assay and antibody binding test, respectively. Serum drug levels were considered positive for infliximab if $>0.035 \mu \mathrm{g} / \mathrm{mL}$, for adalimumab $>0.024 \mu \mathrm{g} / \mathrm{mL}$, for etanercept $>0.035 \mu \mathrm{g} / \mathrm{mL}$, rituximab $>0.75 \mu \mathrm{g} / \mathrm{mL}$, while the cut-offs for ADA positivity to infliximab was established at $5 \mathrm{AU} / \mathrm{ml}$, for adalimumab at $10 \mathrm{AU} / \mathrm{mL}$, etanercept at $142 \mathrm{AU} / \mathrm{mL}$, rituximab $140 \mathrm{AU} / \mathrm{mL}$ (ELISA, Progenika)

Results: 42 patients with paradoxical psoriasis ( 30 with de novo lesions, 18 with palmo-plantar pustulosis, 12 with exacerbation of pre-existent lesions), 12 with paradoxical uveitis ( 5 with a new onset uveitis) and 3 with paradoxical Crohn's were included in the final analysis. Paradoxical events related to abatacept, certolizumab (one de novo psoriasis per agent) or golimumab (2 new onset uveitis, one flare of Chron's disease) were excluded from the final analysis as no immunogenicity lab tests were provided.

Drug induced paradoxical events were described not only with all TNF inhibitors but also with rituximab. DL was in normal range in all these patients; in addition,
ADA were either negative, or, if present, had no impact on drug concentration. Furthermore, we found no significant differences in drug levels of TNF inhibitors for RA, PsA and AS.

Moreover, no statistical significant differences were observed in the detection of ADA between the three groups.

Conclusions: Patients who develop either true or borderline paradoxical $A E$ have adequate drug levels, with normal ADA concentrations.

Disclosure of Interest: None declared

DOI: 10.1136/annrheumdis-2018-eular.4854

\section{FRI0110 THE IMPACT OF SEROPOSITIVITY ON THE EFFECTIVENESS OF ABATACEPT VERSUS TNF INHIBITORS IN RHEUMATOID ARTHRITIS. REAL LIFE DATA FROM SEVERAL EUROPEAN REGISTRIES (THE PAN-ABA STUDY)}

D. Courvoisier ${ }^{1}$, D. Mongin ${ }^{1}$, M. Hetland ${ }^{2}$, K. Pavelka ${ }^{3,4}$, C. Turesson ${ }^{5}$, S.

A. Bergstra ${ }^{6}$, T. K. Kvien ${ }^{7}$, M. J. Santos ${ }^{8}$, V. Hernandez ${ }^{9}$, F. lannone ${ }^{10}$, J.

E. Gottenberg ${ }^{11}$, X. Mariette ${ }^{12}$, S. Kubo ${ }^{13}, Y$. Tanaka ${ }^{13}$, D. Choquette ${ }^{14}$,

R. Ionescu ${ }^{15}$, A. Finckh ${ }^{1} .{ }^{1}$ University Hospitals of Geneva, Geneva, Switzerland, ${ }^{2}$ DANBIO, Rigshospitalet, Denmark, ${ }^{3}$ Institute of Rheumatology, ${ }^{4}$ Rheumatology, First Faculty of Medicine, Charles U, Prague, Czech Republic, ${ }^{5}$ Lund University, Malmö, Sweden, ${ }^{6}$ LUMC, Leiden, Netherlands, ${ }^{7}$ Diakonhjemmet Hospital, Oslo, Norway, ${ }^{8}$ Reuma.pt, Lisbon, Portugal, ${ }^{9}$ Hospital Clinic, Barcelona, Spain,

${ }^{10}$ University of Bari, Bari, Italy, ${ }^{11}$ Chu, Strassbourg, ${ }^{12}$ Paris-Sud University, Le Kremlin-Bicêtre, France, ${ }^{13}$ University of Occupational and Environmental Health, Fukuoka, Japan, ${ }^{14}$ Institut de rhumatologie de Montreal, Chum, U de M, Montreal, Canada, ${ }^{15}$ Department of Internal Medicine and Rheumatology, Sf Maria Hospital, U Medicine and Pharmacy, Bucharest, Romania

Background: Rheumatoid factor (RF) and anti-citrullinated protein antibodies (ACPA) are used as diagnostic tools, but may also be used as prognostic factors, as these biomarkers have been associated with better clinical responses to some biologic agents in rheumatoid arthritis (RA).

Objectives: To compare the impact of seropositivity on drug discontinuation and effectiveness for abatacept (ABA) and TNF inhibitors (TNFi) in patients (pts) with RA.

Methods: Pooled analysis of 13 observational RA registries from countries (FR, IT, CZ, DK, NO, PT, RO, ES, SE, CH, NL, JP, CA) where both ABA and TNFi were available concomitantly. Inclusion criteria were RA diagnosis, treatment with $A B A$ or TNFi, and available RF or ACPA status. Main exposure was seropositivity: positive if RF or ACPA were positive, negative if both were negative, and missing if one was missing and the other was negative. Primary endpoint was drug discontinuation, analysed using Cox models, including treatment, seropositivity, and their interaction, adjusting for patient-, treatment-, and disease-characteristics using strata terms for country and calendar year. We first tested for effect modification by country by additionally including an interaction term between treatment, seropositivity and country. Since we found no effect modification, we took out the interaction term. Effectiveness was analysed using DAS28 remission and low disease activity (LDA) at 1 year, corrected for attrition using Lundex ${ }^{1}$.

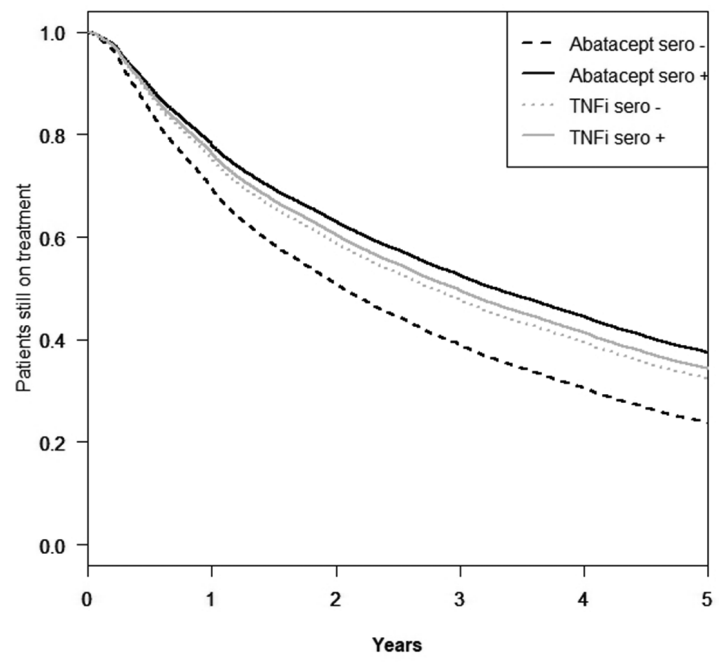

Results: Using data from 39266 treatment-courses, in crude analyses, seronegativity was associated with higher drug discontinuation for pts on ABA but not on TNFi ( $p$ interaction $<0.001$ ), with a hazard ratio $(\mathrm{HR})$ for seropositive vs seronegative of $0.74(95 \% \mathrm{Cl}: 0.66-0.82)$ for pts on $\mathrm{ABA}$ and $0.96(95 \% \mathrm{Cl}: 0.92-1.01)$ for 